\section{COMBINING TIME RESOLVED CONTRAST ENHANCED MR ANGIOGRAPHY WITH VOLUMETRIC HIGH RESOLUTION T2-WEIGHTED IMAGING IS A HIGHLY ACCURATE, NON-INVASIVE METHOD FOR LOCALIZATION OF SPINAL DURAL AV FISTULAS: A CASE SERIES}

M Martucci*, A Oppenheimer, M Moogerfeld, M Obrzut. Neurology, Cleveland Clinic, Weston, FL

\subsection{6/neurintsurg-2020-SNIS. 179}

Objective To describe a non-invasive method for prospectively localizing Spinal Dural AV Fistulas (SDAVF) with contrast enhanced magnetic resonance imaging prior to spinal angiography and treatment,by combining Time Resolved Contrast Enhanced MR Angiography (TRCE-MRA) with Volumetric High Resolution T2-weighted Imaging (VHR-T2I) sequences.

Background The gold standard for the localization of SDAVF is spinal angiography. A complete spinalangiogram involves the sampling of all segmental arteries, which can be time consuming and carries therisks of contrast nephropathy and radiation exposure. A non-invasive method of prospectively localizing the SDAVF feeding segmental arteries would be highly beneficial, as it would allow focusing the spinalangiogram to only a few vessels, decreasing procedure time and mitigating these risks. TRCE-MRA has been utilized in the localization of SDAVFs in the past. This method, however, has been limited by the inherent tradeoff that exists between temporal and spatial resolution in MR imaging. Even on advanced3 Tesla systems, TRCE-MRA of the spine at frame rates higher than 1 per 2 seconds have poor signal tonoise and anatomical detail, making the identification of the small SDAVF feeding vessels a challenge.VHR-T2-I sequences have very high anatomical detail but cannot assess vessel flow. In this case series,we present a method that co-registers the temporal information from TRCE-MRA and the spatial information from VHR-T2-I to prospectively identify the feeding vessels of SDAVFs with a very high accuracy.

Methods Five consecutive patients with clinical and imaging findings consistent with SDAVF underwent 3 Tesla CE-MRA on a Siemens MAGNETOM Skyra system prior to spinal angiography. For each patient, Siemens Time-resolved angiography With Stochastic Trajectories (TWIST) sequences at frame rates of 1 per 2 seconds or higher were obtained. A single slab 3D single slab turbo spin echo (SPACE) sequence was then obtained and the sequences were co-registered. The images were reviewed by aneuroradiologist and the feeding segmental artery to the SDAVF was identified. Subsequently, each patient underwent spinal angiography. In all patients the segmental artery feeding the SDAVF was correctly prospectively identified by the CE-MRA.

Results In all patients in this case series, CE-MRA with TWIST/SPACE co-registration prospectively identified the SDAVF feeding artery. This resulted in a reduction in procedure time, contrast dose and radiation exposure. No procedure complications were encountered. In one patient with advanced atherosclerotic disease, the CE-MRA was critical in identifying the SDAVF feeding artery, as severe stenosis at the origin of the feeding artery made catheterization difficult and the vessel might have beenmissed if not for the prospective information from the CE-MRA.

Conclusion This case series illustrates how combining TRCEMRA and VHR-T2-I can prospectively identify the feeding segmental branches of SDAVFs, resulting in reduced procedure time and risks ofpre-treatment spinal angiography.

Disclosures M. Martucci: None. A. Oppenheimer: None. M. Moogerfeld: None. M. Obrzut: None.

\section{E-148 PIPELINE EMBOLIZATION DEVICE TREATMENT OF INTRACRANIAL ANEURYSMS IN PEDIATRIC PATIENTS: A PATIENT-LEVEL META-ANALYSIS}

N Shlobin*, M Potts. Neurological Surgery, Feinberg School of Medicine, Chicago, IL

\subsection{6/neurintsurg-2020-SNIS. 180}

Introduction While the Pipeline Embolization Device (PED, Medtronic) is safe and effective in the treatment of intracranial aneurysms in adults, less is known about its safety and effectiveness in pediatric patients. We aim to report clinical outcomes in patients aged 18 or younger undergoing flow diversion with PED for intracranial aneurysms.

Methods PubMed, Embase, and Scopus were searched for reports of pediatric patients treated with PED. Disaggregated data was available for 44 pediatric patients with 48 aneurysms. Data regarding demographics, aneurysm characteristics, treatment, follow-up, clinical outcomes, and complications were collected.

Results The average age of patients was $10.5 \pm 4.3$ years, $47.8 \%$ of whom were male, while $34.1 \%$ were female, and the sex of $18.1 \%$ was not reported. A total of 23 aneurysms $(52.2 \%)$ were in the posterior circulation. Fourteen aneurysms (29.2\%) were fusiform, and $12(25.0 \%)$ were dissecting. Fifteen aneurysms (31.3\%) were giant, 14 (29.2\%) were small, and $9(18.9 \%)$ were large. Fourteen aneurysms (29.2\%) presented with mass effect, followed by 11 (22.9\%) with subarachnoid hemorrhage, and $6(12.5 \%)$ each with headache and recurrence. The number of PEDs used for an aneurysm was $2.0 \pm 1.5$, and 10 aneurysms (20.8\%) were treated with adjunct coiling. The average imaging and clinical follow-up for the aneurysms were $10.1 \pm 7.9$ and $10.5 \pm 8.1$ months, respectively. Thirty-four aneurysms (70.8\%) were completely occluded. Four patients (10.0\%) experienced complications, including one death, recurrence, brainstem compression, and in-stent stenosis.

Conclusions PED is safe and effective for intracranial aneurysm treatment in pediatric patients. Proper guidelines regarding use of PED in children should be created.

Disclosures N. Shlobin: None. M. Potts: None.

\section{E-149 THE OUTCOME OF TRANSVENOUS EMBOLIZATION FOR VEIN OF GALEN MALFORMATION AS A LAST PROCEDURE}

T Shigematsu*, A Berenstein. Cerebrovascular Center, Department of Neurosurgery, Mount Sinai Health System, New York, NY

\subsection{6/neurintsurg-2020-SNIS.181}

Backgrounds Vein of Galen aneurysmal malformation (VGAM) is a rare congenital vascular malformation representing $<1 \%$ of all arteriovenous malformations. In the newborn period treatment is done for untreatable CHF. There have been reports of transvenous coil embolization either trans-tuorqualar, or tran-femoral, although effective in controlling the high output failure, the results are less than optimal, and has lost 